\title{
Universal Decoding for Arbitrary Channels Relative to a Given Class of Decoding Metrics
}

\author{
Neri Merhav \\ Department of Electrical Engineering \\ Technion - Israel Institute of Technology \\ Technion City, Haifa 32000, ISRAEL \\ E-mail: merhav@ee.technion.ac.il
}

\begin{abstract}
We consider the problem of universal decoding for arbitrary unknown channels in the random coding regime. For a given random coding distribution and a given class of metric decoders, we propose a generic universal decoder whose average error probability is, within a sub-exponential multiplicative factor, no larger than that of the best decoder within this class of decoders. Since the optimum, maximum likelihood (ML) decoder of the underlying channel is not necessarily assumed to belong to the given class of decoders, this setting suggests a common generalized framework for: (i) mismatched decoding, (ii) universal decoding for a given family of channels, and (iii) universal coding and decoding for deterministic channels using the individual-sequence approach. The proof of our universality result is fairly simple, and it is demonstrated how some earlier results on universal decoding are obtained as special cases. We also demonstrate how our method extends to more complicated scenarios, like incorporation of noiseless feedback, and the multiple access channel.
\end{abstract}

Index Terms: Universal decoding, mismatched decoding, error exponents, finite-state machines, Lempel-Ziv algorithm, feedback, multiple access channel. 


\section{Introduction}

In many situations practically encountered in coded communication systems, channel uncertainty and variability preclude the implementation of the optimum maximum likelihood (ML) decoder, and so, universal decoders, independent of the unknown channel, are sought.

The topic of universal coding and decoding under channel uncertainty has received very much attention in the last four decades. In [7], Goppa offered the maximum mutual information (MMI) decoder, which decides in favor of the codeword having the maximum empirical mutual information with the channel output sequence. Goppa showed that for discrete memoryless channels (DMC's), MMI decoding achieves capacity. Csiszár and Körner [3] have also studied the problem of universal decoding for DMC's with finite input and output alphabets. They showed that the random coding error exponent of the MMI decoder, associated with a uniform random coding distribution over a certain type class, achieves the optimum random coding error exponent. Csiszár [2] proved that for any modulo-additive DMC and the uniform random coding distribution over linear codes, the optimum random coding error exponent is universally achieved by a decoder that minimizes the empirical entropy of the difference between the output sequence and the input sequence. In [13] an analogous result was derived for a certain parametric class of memoryless Gaussian channels with an unknown interference signal.

In the realm of channels with memory, Ziv [21] explored the universal decoding problem for unknown finite-state channels with finite input and output alphabets, for which the next channel state is a deterministic unknown function (a.k.a. the next-state function) of the channel current state and current inputs and outputs. For codes governed by uniform random coding over a given set, he proved that a decoder based on the Lempel-Ziv algorithm asymptotically achieves the error exponent associated with ML decoding. In [9], Lapidoth and Ziv proved that the latter decoder continues to be universally asymptotically optimum in the random coding error exponent sense even for a wider class of finite-state channels, namely, those with stochastic, rather than deterministic, next-state functions. In [5], Feder and Lapidoth furnished sufficient conditions for families of channels with memory to have universal decoders that asymptotically achieve the random coding error exponent associated with ML decoding. In [6], Feder and Merhav proposed a competitive minimax criterion, in an effort to develop a more general systematic approach to the problem of 
universal decoding. According to this approach, an optimum decoder is sought in the quest for minimizing (over all decision rules) the maximum (over all channels in the family) ratio between the error probability associated with a given channel and a given decision rule, and the error probability of the ML decoder for that channel, possibly raised some power less than unity.

More recently, interesting attempts (see, e.g., [11], [12], [16], [18]) were made to devise coding and decoding strategies that avoid any probabilistic assumptions concerning the operation of the channel. This is in the spirit of the individual-sequence approach in information theory, that was originally developed in universal source coding [22] and later on further exercised in other problem areas. In [11], the notion of empirical rate functions has been established and investigated (with and without feedback) for a given input distribution and for given posterior probability function (or a family of such functions) of the channel input sequence given the output sequence. In [16], capacity-achieving (or "porosity-achieving", in the terminology of [16]) universal encoders and decoders, namely, encoder-decoder pairs with coding rates as high as the best finite-state encoder and decoder, were devised for modulo additive channels with deterministic noise sequences and noiseless feedback. This feedback is necessary to let the encoder adapt to the channel, which otherwise does not access the channel output and thus cannot learn (either implicitly or explicitly) the characteristics of the channel.

In this paper, we take a somewhat different approach. We consider the problem of universal decoding for arbitrary unknown channels in the random coding regime. For a given random coding distribution and a given class of metric decoders, we propose a generic universal decoder whose average error probability is, within a sub-exponential multiplicative factor, no larger than that of the best decoder in this class of decoders. Since the optimum, ML decoder of the underlying channel is not necessarily assumed to belong to the given class of decoders, this setting is suitable as a common ground for:

1. Mismatched decoding (see, e.g., [4], [8], [15]) - when the reference class of decoders is a singleton and the ML decoder for the underlying channel is different from the unique decoder in this singleton.

2. Universal decoding for a given family of channels (as in papers cited in the second and third paragraphs above) - when the ML decoder for the underlying channel belongs to the given 
class of decoders.

3. Universal coding and decoding for deterministic channels using the individual-sequence approach (as in [11], [12], [16], [18]) - when the underlying channel is deterministic and the universality is relative to a given class of coding/decoding strategies.

The proof of our universality result is fairly simple and general, and it is demonstrated how some earlier mentioned results on universal decoding are obtained as special cases. It is based on very simple upper and lower bounds on the probabilities of the pairwise error events, as well as on a lower bound due to Shulman [19, Lemma A.2] on the probability of the union of pairwise independent events, which coincides with the union bound up to a factor of $1 / 2$.

Finally, we demonstrate how our method extends to more complicated scenarios. The first extension corresponds to random coding distributions that allow to incorporate noiseless feedback. This extension is fairly straightforward, but its main importance is in allowing adaptation of the random coding distribution to the channel statistical characteristics. The second extension is to the problem of universal decoding for multiple access channels (MAC's) with respect to a given class of decoding metrics. This extension is not trivial since the universal decoding metric has to confront three different types of error events (in the case of a MAC with two senders). In particular, it turns out that the resulting universal decoding metric is surprisingly different from those of earlier works on universal decoding for the MAC [10], [5, Section VIII], [17], mostly because the problem setting here is different from those of these earlier works (in the sense that the universality here is relative to a given class of decoders while the underlying channel is arbitrary, and not relative to a given class of channels).

The outline of the paper is as follows. In Section 2, we establish notation conventions and we formalize the problem setting. Section 3 contains our main result and its proof, as well as a discussion and examples. Section 4 suggests guidelines for approximating the universal decoding metric in situations where it is hard to compute, and thereby shows how Ziv's decoding metric [21] falls within our framework. Finally, in Section 5, we provide extensions to the case where feedback is available and the case of the MAC. 


\section{Notation Conventions and Problem Formulation}

\subsection{Notation Conventions}

Throughout this paper, scalar random variables (RV's) are denoted by capital letters, their sample values are denoted by the respective lower case letters, and their alphabets are denoted by the respective calligraphic letters. A similar convention applies to random vectors of dimension $n$ and their sample values, which will be denoted with same symbols in the bold face font. The set of all $n$-vectors with components taking values in a certain alphabet, will be denoted as the same alphabet superscripted by $n$. Sources and channels will be denoted generically by the letter $P$ or $Q$. For example, the channel input probability distribution function will be denoted by $Q(\boldsymbol{x}), \boldsymbol{x} \in \mathcal{X}^{n}$, and the conditional probability distribution of the channel output vector $\boldsymbol{y} \in \mathcal{Y}^{n}$ given the input vector $\boldsymbol{x} \in \mathcal{X}^{n}$, will be denoted by $P(\boldsymbol{y} \mid \boldsymbol{x})$. Information theoretic quantities like entropies and conditional entropies, will be denoted following the standard conventions of the information theory literature, e.g., $H(\boldsymbol{X}), H(\boldsymbol{X} \mid \boldsymbol{Y})$, etc. The expectation operator will be denoted by $\boldsymbol{E}\{\cdot\}$ and the cardinality of a finite set $\mathcal{A}$ will be denoted by $|\mathcal{A}|$.

For a given sequence $\boldsymbol{x} \in \mathcal{X}^{n}, \mathcal{X}$ being a finite alphabet, $\hat{P}_{\boldsymbol{x}}$ denotes the empirical distribution on $\mathcal{X}$ extracted from $\boldsymbol{x}$, in other words, $\hat{P}_{\boldsymbol{x}}$ is the vector $\left\{\hat{P}_{\boldsymbol{x}}(x), x \in \mathcal{X}\right\}$, where $\hat{P}_{\boldsymbol{x}}(x)$ is the relative frequency of the letter $x$ in the vector $\boldsymbol{x}$. The type class of $\boldsymbol{x}$, denoted $T_{\boldsymbol{x}}$, is the set of all sequences $\boldsymbol{x}^{\prime} \in \mathcal{X}^{n}$ with $\hat{P}_{\boldsymbol{x}^{\prime}}=\hat{P} \boldsymbol{x}$. Similarly, for a pair of sequences $(\boldsymbol{x}, \boldsymbol{y}) \in \mathcal{X}^{n} \times \mathcal{Y}^{n}$, the empirical distribution $\hat{P}_{\boldsymbol{x} \boldsymbol{y}}$ is the matrix of relative frequencies $\left\{\hat{P}_{\boldsymbol{x} \boldsymbol{y}}(x, y), x \in \mathcal{X}, y \in \mathcal{Y}\right\}$ and the type class $T_{\boldsymbol{x} \boldsymbol{y}}$ is the set of pairs $\left(\boldsymbol{x}^{\prime}, \boldsymbol{y}^{\prime}\right) \in \mathcal{X}^{n} \times \mathcal{Y}^{n}$ with $\hat{P}_{\boldsymbol{x}^{\prime} \boldsymbol{y}^{\prime}}=\hat{P}_{\boldsymbol{x} \boldsymbol{y}}$. For a given $\boldsymbol{y}$, $T_{\boldsymbol{x}} \mid \boldsymbol{y}$ denotes the conditional type class of $\boldsymbol{x}$ given $\boldsymbol{y}$, which is the set of vectors $\left\{\boldsymbol{x}^{\prime}\right\}$ such that $\left(\boldsymbol{x}^{\prime}, \boldsymbol{y}\right) \in T_{\boldsymbol{x} \boldsymbol{y}}$. Information measures induced by empirical distributions, i.e., empirical information measures, will be denoted with a hat and a subscript that indicates the sequence(s) from which they are induced. For example, $\hat{H}_{\boldsymbol{x}}(X)$ is the empirical entropy extracted from $\boldsymbol{x} \in \mathcal{X}^{n}$, namely, the entropy of a random variable $X$ whose distribution is $\hat{P}_{\boldsymbol{x}}$. Similarly, $\hat{H}_{\boldsymbol{x} \boldsymbol{y}}(X \mid Y)$ and $\hat{I}_{\boldsymbol{x} \boldsymbol{y}}(X ; Y)$ are, respectively, the empirical conditional entropy of $X$ given $Y$, and the empirical mutual information between $X$ and $Y$, extracted from $(\boldsymbol{x}, \boldsymbol{y})$, and so on.

For two sequences of positive numbers, $\left\{a_{n}\right\}$ and $\left\{b_{n}\right\}$, the notation $a_{n} \doteq b_{n}$ means that

$\frac{1}{n} \log \frac{a_{n}}{b_{n}} \rightarrow 0$ as $n \rightarrow \infty$. Similarly, $a_{n} \leq b_{n}$ means that $\lim \sup _{n \rightarrow \infty} \frac{1}{n} \log \frac{a_{n}}{b_{n}} \leq 0$, and so on. 
The functions $\log (\cdot)$ and $\exp (\cdot)$, throughout this paper, will be defined to the base 2 , unless otherwise indicated. The operation $[\cdot]_{+}$will mean positive clipping, that is $[x]_{+}=\max \{0, x\}$.

\subsection{Problem Formulation}

Consider a random selection of a codebook $\mathcal{C}=\left\{\boldsymbol{x}_{1}, \ldots, \boldsymbol{x}_{M}\right\} \subseteq \mathcal{X}^{n}$, where $M=2^{n R}, R$ being the coding rate in bits per channel use. The marginal probability distribution function of each codeword $\boldsymbol{x}_{i}$ is denoted by $Q\left(\boldsymbol{x}_{i}\right)$. It will be assumed that the various codewords are pairwise independent. ${ }^{1}$ Let $P(\boldsymbol{y} \mid \boldsymbol{x})$ be the conditional probability distribution of the channel output vector $\boldsymbol{y} \in \mathcal{Y}^{n}$ given the channel input vector $\boldsymbol{x} \in \mathcal{X}^{n}$. We make no assumptions at all concerning the channel. ${ }^{2}$ We will assume, throughout most of this paper, that both the channel input alphabet $\mathcal{X}$ and the channel output alphabet $\mathcal{Y}$ are finite sets. Finally, we define a class of decoding metrics, as a class of real functions, $\mathcal{M}=\left\{m_{\theta}(\boldsymbol{x}, \boldsymbol{y}), \theta \in \Theta, \boldsymbol{x} \in \mathcal{X}^{n}, \boldsymbol{y} \in \mathcal{Y}^{n}\right\}$, where $\Theta$ is an index set, which may be either finite, countably infinite, or uncountably infinite. ${ }^{3}$ The decoder associated with the decoding metric $m_{\theta}$, which will be denoted by $\mathcal{D}_{\theta}$, decides in favor of the message $i \in\{1, \ldots, M\}$ which maximizes $m_{\theta}\left(\boldsymbol{x}_{i}, \boldsymbol{y}\right)$ for the given received channel output vector $\boldsymbol{y}$, that is

$$
\mathcal{D}_{\theta}: \quad \hat{i}=\operatorname{argmax}_{1 \leq i \leq M} m_{\theta}\left(\boldsymbol{x}_{i}, \boldsymbol{y}\right) .
$$

The message $i$ is assumed to be uniformly distributed in the set $\{1,2, \ldots, M\}$. It should be emphasized that the optimum, ML decoding metric for the underlying channel $P(\boldsymbol{y} \mid \boldsymbol{x})$, may not necessarily belong to the given class of decoding metrics $\mathcal{M}$. In other words, this is a problem of universal decoding with possible mismatch.

The average error probability $\bar{P}_{e, \theta}(R, n)$, associated with the decoder $\mathcal{D}_{\theta}$, is defined as

$$
\bar{P}_{e, \theta}(R, n) \triangleq \frac{1}{M} \sum_{i=1}^{M} \operatorname{Pr} \bigcup_{j \neq i}\left\{m_{\theta}\left(\boldsymbol{X}_{j}, \boldsymbol{Y}\right) \geq m_{\theta}\left(\boldsymbol{X}_{i}, \boldsymbol{Y}\right) \mid \boldsymbol{X}_{i} \text { sent }\right\},
$$

where $\operatorname{Pr}\{\cdot\}$ designates the probability measure pertaining to the randomness of the codebook $\mathcal{C}$ as well as that of the channel output given its input.

\footnotetext{
${ }^{1}$ Full independence of all codewords is allowed, but not enforced. This permits our setting to include, among other things, ensembles of linear codes, which are well known to admit pairwise independence, but not stronger notions of independence.

${ }^{2}$ We even allow a deterministic channel, which puts all its probabilistic mass on one vector $\boldsymbol{y}$ which is given by a deterministic function of $\boldsymbol{x}$.

${ }^{3}$ For example, in the uncountably infinite case, $\theta$ may designate a parameter and $\left\{m_{\theta}(\boldsymbol{x}, \boldsymbol{y}), \theta \in \Theta\right\}$ may be a smooth parametric family.
} 
While the decoder $\mathcal{D}_{\theta}$, that minimizes $\bar{P}_{e, \theta}(R, n)$ within the class, depends, in general, on the unknown underlying channel $P(\boldsymbol{y} \mid \boldsymbol{x})$, our goal is to devise a universal decoder $\mathcal{U}$, with a decoding metric $U(\boldsymbol{x}, \boldsymbol{y})$, independent of the underlying channel $P(\boldsymbol{y} \mid \boldsymbol{x})$, whose average error probability would be essentially as small as $\min _{\theta} \bar{P}_{e, \theta}(R, n)$, whatever the underlying channel may be. By "essentially as small", we mean that the average error probability associated with the universal decoder,

$$
\bar{P}_{e, u}(R, n) \triangleq \frac{1}{M} \sum_{i=1}^{M} \operatorname{Pr} \bigcup_{j \neq i}\left\{U\left(\boldsymbol{X}_{j}, \boldsymbol{Y}\right) \geq U\left(\boldsymbol{X}_{i}, \boldsymbol{Y}\right) \mid \boldsymbol{X}_{i} \text { sent }\right\},
$$

would not exceed $\min _{\theta} \bar{P}_{e, \theta}(R, n)$ by more than a multiplicative factor that grows sub-exponentially with $n$. This means that whenever $\min _{\theta} \bar{P}_{e, \theta}(R, n)$ decays exponentially with $n$, then so does $\bar{P}_{e, u}(R, n)$, and at an exponential rate at least as fast. Another (essentially equivalent) legitimate goal is that $\bar{P}_{e, u}(R, n)$ would not be larger than $\min _{\theta} \bar{P}_{e, \theta}\left(R+\Delta_{n}, n\right)$, where $\Delta_{n} \rightarrow 0$ as $n \rightarrow \infty$. In the next section, we shall see that both goals are met by a conceptually simple universal decoding metric $U(\boldsymbol{x}, \boldsymbol{y})$, which depends solely on $Q$ and on the reference class $\mathcal{M}$ of competing decoding metrics.

\section{Main Result}

Consider the given random coding distribution $Q$ and the given family of decoding metrics $\mathcal{M}=$ $\left\{m_{\theta}(\boldsymbol{x}, \boldsymbol{y}), \theta \in \Theta\right\}$, as defined earlier. Let us define

$$
\mathcal{T}(\boldsymbol{x} \mid \boldsymbol{y}) \triangleq\left\{\boldsymbol{x}^{\prime}: \quad \forall \theta \in \Theta \quad m_{\theta}\left(\boldsymbol{x}^{\prime}, \boldsymbol{y}\right)=m_{\theta}(\boldsymbol{x}, \boldsymbol{y})\right\}
$$

Our universal decoding metric is defined as

$$
U(\boldsymbol{x}, \boldsymbol{y}) \triangleq-\frac{1}{n} \log Q[\mathcal{T}(\boldsymbol{x} \mid \boldsymbol{y})] .
$$

Note that when $\mathcal{X}$ is a discrete alphabet, $\{\mathcal{T}(\boldsymbol{x} \mid \boldsymbol{y})\}$ are equivalence classes for every $\boldsymbol{y} \in \mathcal{Y}^{n}$, and so the space $\mathcal{X}^{n}$ can be partitioned into a disjoint union of them. Let $K_{n}(\boldsymbol{y})$ denote the number of equivalence classes $\{\mathcal{T}(\boldsymbol{x} \mid \boldsymbol{y})\}$ for a given $\boldsymbol{y}$. Also define

$$
K_{n} \triangleq \max _{\boldsymbol{y} \in \mathcal{Y}^{n}} K_{n}(\boldsymbol{y})
$$

and

$$
\Delta_{n} \triangleq \frac{\log K_{n}}{n} .
$$


Our main result is the following theorem:

Theorem 1 Under the assumptions of Section 2, the universal decoding metric defined in eq. (5) satisfies:

$$
\bar{P}_{e, u}(R, n) \leq 2 \cdot 2^{n \Delta_{n}} \cdot \min _{\theta \in \Theta} \bar{P}_{e, \theta}(R, n)
$$

and

$$
\bar{P}_{e, u}(R, n) \leq 2 \cdot \min _{\theta \in \Theta} \bar{P}_{e, \theta}\left(R+\Delta_{n}, n\right)
$$

Discussion. The theorem is, of course, meaningful when $\Delta_{n} \rightarrow 0$ as $n \rightarrow \infty$, which means that the number of various equivalence classes $\{\mathcal{T}(\boldsymbol{x} \mid \boldsymbol{y})\}$ grows sub-exponentially as a function of $n$, uniformly in $\boldsymbol{y}$. As mentioned earlier, in this case, whenever $\min _{\theta \in \Theta} \bar{P}_{e, \theta}(R, n)$ decays exponentially with $n$, then $\bar{P}_{e, u}(R, n)$ decays exponentially as well, and at least as fast. Consequently, the maximum information rate pertaining to the universal decoder is at least as large as that of the best decoder $\mathcal{D}_{\theta}$ in the given class. We therefore learn from Theorem 1 that a sufficient condition for the existence of a universal decoder is $\lim _{n \rightarrow \infty} \Delta_{n}=0$. Whether this is also a necessary condition, remains an open question at this point. Necessary and sufficient conditions for universality in the ordinary setting have been furnished in [5] and [6].

Intuitively, the behavior of $\Delta_{n}$ for large $n$ is a measure of the richness of the class of decoding metrics. The larger is the index set $\Theta$, the smaller are the equivalence classes $\{\mathcal{T}(\boldsymbol{x} \mid \boldsymbol{y})\}$, and then their total number $K_{n}(\boldsymbol{y})$ becomes larger, and so does $\Delta_{n}$. Universality is enabled, using this method, as long as the set $\Theta$ is not too rich, so that $\Delta_{n}$ still vanishes as $n$ grows without bound.

When $Q$ is invariant within $\mathcal{T}(\boldsymbol{x} \mid \boldsymbol{y})$ (i.e., $\boldsymbol{x}^{\prime} \in \mathcal{T}(\boldsymbol{x} \mid \boldsymbol{y})$ implies $Q\left(\boldsymbol{x}^{\prime}\right)=Q(\boldsymbol{x})$ ), we have

$$
\begin{aligned}
U(\boldsymbol{x}, \boldsymbol{y}) & =-\frac{1}{n} \log Q[\mathcal{T}(\boldsymbol{x} \mid \boldsymbol{y})] \\
& =-\frac{1}{n} \log [Q(\boldsymbol{x}) \cdot|\mathcal{T}(\boldsymbol{x} \mid \boldsymbol{y})|] \\
& =-\frac{1}{n}[\log Q(\boldsymbol{x})+\log |\mathcal{T}(\boldsymbol{x} \mid \boldsymbol{y})|]
\end{aligned}
$$

The choice of a distribution $Q$ that is invariant within $T(\boldsymbol{x} \mid \boldsymbol{y})$ is convenient, because in most cases it is easier to evaluate the $\log$-cardinality of $\mathcal{T}(\boldsymbol{x} \mid \boldsymbol{y})$ (or its $\log$-volume, in the continuous case) than to evaluate its probability under a general probability measure $Q$. 
Before we turn to the proof of Theorem 1, it would be instructive to consider two simple examples. In both of them (as well as in other examples in the sequel) $Q$ is invariant within $\mathcal{T}(\boldsymbol{x} \mid \boldsymbol{y})$.

Example 1. Let $Q$ be the uniform distribution across a single type class, $T_{\boldsymbol{x}}$, and let $\mathcal{M}$ be the class of additive decoding metrics

$$
m_{\theta}(\boldsymbol{x}, \boldsymbol{y})=\sum_{i=1}^{n} \theta\left(x_{i}, y_{i}\right),
$$

where $\{\theta(x, y), x \in \mathcal{X}, y \in \mathcal{Y}\}$ are arbitrary real-valued matrices. In this case, $\mathcal{T}(\boldsymbol{x} \mid \boldsymbol{y})=T_{\boldsymbol{x}} \mid \boldsymbol{y}$, the conditional type class of $\boldsymbol{x}$ given $\boldsymbol{y}$. Since the number of distinct conditional type classes is polynomial in $n$, then $\Delta_{n}$ is proportional to $(\log n) / n$. In this case, we have

$$
\begin{aligned}
U(\boldsymbol{x}, \boldsymbol{y}) & =-\frac{1}{n} \log Q\left[T_{\boldsymbol{x} \mid \boldsymbol{y}}\right] \\
& =-\frac{1}{n} \log \left[Q(\boldsymbol{x}) \cdot \mid T_{\boldsymbol{x}|\boldsymbol{y}|]}\right. \\
& =\hat{H}_{\boldsymbol{x}}(X)-\hat{H}_{\boldsymbol{x} \boldsymbol{y}}(X \mid Y)+o(n) \\
& =\hat{I}_{\boldsymbol{x} \boldsymbol{y}}(X ; Y)+o(n) .
\end{aligned}
$$

and so, the proposed universal decoder essentially ${ }^{4}$ coincides with the MMI decoder. However, since $\mathcal{C}$ is a constant composition code, under this particular choice of $Q, \hat{H}_{\boldsymbol{x}_{i}}(X)$ is the same for all $i$, and so, this decoder is equivalent to the decoder that selects the codeword that minimizes the empirical conditional entropy of $X$ given $Y$, namely, $\min _{i} \hat{H}_{\boldsymbol{x}_{i} \boldsymbol{y}}(X \mid Y)$. If, on the other hand, $Q$ is an i.i.d. probability distribution function, namely, $Q(\boldsymbol{x})=\prod_{i=1}^{n} Q\left(x_{i}\right)$, then the universal decoding metric becomes

$$
U(\boldsymbol{x}, \boldsymbol{y})=\hat{I}_{\boldsymbol{x} \boldsymbol{y}}(X ; Y)+D\left(\hat{P}_{\boldsymbol{x}} \| Q\right)+o(n),
$$

where $D\left(\hat{P}_{\boldsymbol{x}} \| Q\right)$ is the Kullback-Leibler divergence between $\hat{P}_{\boldsymbol{x}}$ and $Q$.

For certain classes of channels (e.g., arbitrarily varying channels), it is not difficult to derive single-letter formulas for the maximum achievable information rates in the random coding regime, that is, the supremum of $R$ such that $\bar{P}_{e, u}(R, n) \rightarrow 0$ as $n \rightarrow \infty$. The main tool for this purpose is the method of types. This concludes Example 1.

\footnotetext{
${ }^{4}$ The $o(n)$ term can be omitted with affecting the asymptotic performance.
} 
Example 2. Let $\mathcal{X}=\mathcal{Y}=\mathbb{R}$ and let

$$
Q(\boldsymbol{x})=\frac{e^{-\sum_{i=1}^{n} x_{i}^{2} /\left(2 \sigma^{2}\right)}}{\left(2 \pi \sigma^{2}\right)^{n / 2}} .
$$

Let $\theta=\left(\theta_{1}, \theta_{2}\right) \in \mathbb{R}^{2}$ and $\mathcal{M}$ be the class of decoding metrics of the form ${ }^{5}$

$$
m_{\theta}(\boldsymbol{x}, \boldsymbol{y})=\theta_{1} \sum_{i=1}^{n} x_{i} y_{i}+\theta_{2} \sum_{i=1}^{n} x_{i}^{2} .
$$

In principle, $\mathcal{T}(\boldsymbol{x} \mid \boldsymbol{y})$ is the set of all $\left\{\boldsymbol{x}^{\prime}\right\}$ with the same empirical power and the same empirical correlation with $\boldsymbol{y}$, as those of $\boldsymbol{x}$. However, since in this example the sequences $\boldsymbol{x}$ and $\boldsymbol{y}$ have continuous-valued components, some tolerance must be allowed in the empirical correlation $C(\boldsymbol{x}, \boldsymbol{y})=\frac{1}{n} \sum_{i=1}^{n} x_{i} y_{i}$ and empirical power, $S(\boldsymbol{x})=\frac{1}{n} \sum_{i=1}^{n} x_{i}^{2}$, for $\mathcal{T}(\boldsymbol{x} \mid \boldsymbol{y})$ to have positive probability (and positive volume), and so, $\mathcal{T}(\boldsymbol{x} \mid \boldsymbol{y})$ should be redefined as the set of sequences $\boldsymbol{x}^{\prime}$, where $C\left(\boldsymbol{x}^{\prime}, \boldsymbol{y}\right)$ and $S\left(\boldsymbol{x}^{\prime}\right)$ are within $\epsilon(\epsilon>0$, but small) close to $C(\boldsymbol{x}, \boldsymbol{y})$ and $S(\boldsymbol{x})$, respectively. Using the methods developed in $[13],{ }^{6}$ it is not difficult to show that, after omitting some additive constants (which do not affect the decision rule), we have in this case

$$
U(\boldsymbol{x}, \boldsymbol{y})=\frac{S(\boldsymbol{x})}{2 \sigma^{2}}-\frac{1}{2} \ln \left[S(\boldsymbol{x})\left(1-\rho_{\boldsymbol{x} \boldsymbol{y}}^{2}\right)\right]
$$

where $\rho \boldsymbol{x y}=C(\boldsymbol{x}, \boldsymbol{y}) / \sqrt{S(\boldsymbol{x}) S(\boldsymbol{y})}$ is the empirical correlation coefficient between $\boldsymbol{x}$ and $\boldsymbol{y}$, and where we have used natural logarithms instead of base 2 logarithms for obvious reasons. The first term stems from $-\frac{1}{n} \ln Q(\boldsymbol{x})$ and the second term comes from the negative log-volume of $\mathcal{T}(\boldsymbol{x} \mid \boldsymbol{y})$. This concludes Example 2.

Proof of Theorem 1. The pairwise average error probability, associated with $m_{\theta}$ is lower bounded by

$$
\begin{aligned}
\bar{\Pi}_{e, \theta}(\boldsymbol{x}, \boldsymbol{y}) & \triangleq \sum_{\left\{\boldsymbol{x}^{\prime}: m_{\theta}\left(\boldsymbol{x}^{\prime}, \boldsymbol{y}\right) \geq m_{\theta}(\boldsymbol{x}, \boldsymbol{y})\right\}} Q\left(\boldsymbol{x}^{\prime}\right) \\
& \geq \sum_{\boldsymbol{x}^{\prime} \in \mathcal{T}(\boldsymbol{x} \mid \boldsymbol{y})} Q\left(\boldsymbol{x}^{\prime}\right) \\
& =Q[\mathcal{T}(\boldsymbol{x} \mid \boldsymbol{y})] \\
& =\exp [-n U(\boldsymbol{x}, \boldsymbol{y})] .
\end{aligned}
$$

\footnotetext{
${ }^{5}$ This class of decoders is clearly motivated by the family of channels $y_{t}=a x_{t}+z_{t}$, where $a$ is an unknown parameter and $z_{t}$ is an i.i.d. Gaussian process, independent of $x_{t}$.

${ }^{6}$ The details are conceptually simple but technically tedious. The interested reader is referred to [13] for a rigorous treatment.
} 
On the other hand, the pairwise error probability associated with the decoding metric $U$ is upper bounded by

$$
\begin{aligned}
\bar{\Pi}_{e, u}(\boldsymbol{x}, \boldsymbol{y}) & \triangleq \sum_{\left\{\boldsymbol{x}^{\prime}: U\left(\boldsymbol{x}^{\prime}, \boldsymbol{y}\right) \geq U(\boldsymbol{x}, \boldsymbol{y})\right\}} Q\left(\boldsymbol{x}^{\prime}\right) \\
& =\sum_{\left\{\mathcal{T}\left(\boldsymbol{x}^{\prime} \mid \boldsymbol{y}\right): U\left(\boldsymbol{x}^{\prime}, \boldsymbol{y}\right) \geq U(\boldsymbol{x}, \boldsymbol{y})\right\}} \sum_{\left\{\boldsymbol{x}_{\boldsymbol{x}} \in \mathcal{T}\left(\boldsymbol{x}^{\prime} \mid \boldsymbol{y}\right)\right.} Q(\tilde{\boldsymbol{x}}) \\
& =\sum_{\left\{\mathcal{T}\left(\boldsymbol{x}^{\prime} \mid \boldsymbol{y}\right): U\left(\boldsymbol{x}^{\prime}, \boldsymbol{y}\right) \geq U(\boldsymbol{x}, \boldsymbol{y})\right\}} Q\left[\mathcal{T}\left(\boldsymbol{x}^{\prime} \mid \boldsymbol{y}\right)\right] \\
& =\sum_{\left\{\mathcal{T}\left(\boldsymbol{x}^{\prime} \mid \boldsymbol{y}\right): U\left(\boldsymbol{x}^{\prime}, \boldsymbol{y}\right) \geq U(\boldsymbol{x}, \boldsymbol{y})\right\}} \exp \left[-n U\left(\boldsymbol{x}^{\prime}, \boldsymbol{y}\right)\right] \\
\leq & \sum_{\left\{\mathcal{T}\left(\boldsymbol{x}^{\prime} \mid \boldsymbol{y}\right): U\left(\boldsymbol{x}^{\prime}, \boldsymbol{y}\right) \geq U(\boldsymbol{x}, \boldsymbol{y})\right\}} \exp [-n U(\boldsymbol{x}, \boldsymbol{y})] \\
\leq & \sum_{\left\{\mathcal{T}\left(\boldsymbol{x}^{\prime} \mid \boldsymbol{y}\right)\right\}} \exp [-n U(\boldsymbol{x}, \boldsymbol{y})] \\
\leq & 2^{n \Delta_{n}} \exp [-n U(\boldsymbol{x}, \boldsymbol{y})] \\
& =\exp \left\{-n\left[U(\boldsymbol{x}, \boldsymbol{y})-\Delta_{n}\right]\right\}
\end{aligned}
$$

where in the second equality we have used the fact that $U(\boldsymbol{x}, \boldsymbol{y})$ depends on $\boldsymbol{x}$ and $\boldsymbol{y}$ only via $\mathcal{T}(\boldsymbol{x} \mid \boldsymbol{y})$ and the last inequality follows from the fact that the number of different equivalence classes $\left\{\mathcal{T}\left(\boldsymbol{x}^{\prime} \mid \boldsymbol{y}\right)\right\}$ is upper bounded by $K_{n}=2^{n \Delta_{n}}$ by definition. Now, as is well known, given $\boldsymbol{x}$ and $\boldsymbol{y}$, the average probability of error can be upper bounded in terms of the average pairwise error probability by the expectation of the union bound, clipped to unity, that is

$$
\bar{P}_{e, u}(R, n) \leq \boldsymbol{E}\left[\min \left\{1,2^{n R} \bar{\Pi}_{e, u}(\boldsymbol{X}, \boldsymbol{Y})\right\}\right] \leq \boldsymbol{E}\left[\min \left\{1,2^{n R} \exp \left(-n\left[U(\boldsymbol{X}, \boldsymbol{Y})-\Delta_{n}\right]\right)\right\}\right]
$$

where the expectation is w.r.t. the randomness of $\boldsymbol{X}$ and $\boldsymbol{Y}$, whose joint distribution is given by $Q(\boldsymbol{x}) P(\boldsymbol{y} \mid \boldsymbol{x})$.

Next, we need a lower bound on $\bar{P}_{e, \theta}(R, n)$ in terms of $\Pi_{e, \theta}(\boldsymbol{x}, \boldsymbol{y})$. To this end, we invoke the following lower bound on the probability of the union of pairwise independent events $\mathcal{A}_{1}, \ldots, \mathcal{A}_{M}$, proved by Shulman $[19 \text {, p. 109, Lemma A.2 }]^{7}$

$$
\operatorname{Pr}\left\{\bigcup_{i=1}^{M} \mathcal{A}_{i}\right\} \geq \frac{1}{2} \cdot \min \left\{1, \sum_{i=1}^{M} \operatorname{Pr}\left(\mathcal{A}_{i}\right)\right\} .
$$

\footnotetext{
${ }^{7} \mathrm{~A}$ similar result was proved independently in [20, Lemma 1] for fully independent events with equal probabilities.
} 
In our case, for a given $\boldsymbol{x}_{i}=\boldsymbol{x}$ and $\boldsymbol{y}$, the events $\left\{m_{\theta}\left(\boldsymbol{X}_{j}, \boldsymbol{y}\right) \geq m_{\theta}(\boldsymbol{x}, \boldsymbol{y})\right\}_{j \neq i}$ are pairwise independent since we have assumed that the various codewords are pairwise independent. Thus, after taking the expectation w.r.t. the joint distribution of $(\boldsymbol{X}, \boldsymbol{Y})$, we have

$$
\bar{P}_{e, \theta}(R, n) \geq \frac{1}{2} \cdot \boldsymbol{E}\left[\min \left\{1,2^{n R} \bar{\Pi}_{e, \theta}(\boldsymbol{X}, \boldsymbol{Y})\right\}\right] \geq \frac{1}{2} \cdot \boldsymbol{E}\left[\min \left\{1,2^{n R} \exp (-n U(\boldsymbol{X}, \boldsymbol{Y})\}\right]\right.
$$

Comparing now the right-most side of eq. (32) with that of eq. (34), we readily see that $\bar{P}_{e, u}(R, n)$ is upper bounded both by $2 \bar{P}_{e, \theta}\left(R+\Delta_{n}, n\right)$ and by $2 \cdot 2^{n \Delta_{n}} \bar{P}_{e, \theta}(R, n)$. The first upper bound is obtained by combining $\Delta_{n}$ and $R$ in (32) and the second upper bound is obtained similarly, by upper bounding the unity term (in $\min \left\{1,2^{n\left[R+\Delta_{n}\right]} \bar{\Pi}_{e, \theta}(\boldsymbol{X}, \boldsymbol{Y})\right\}$ ) by $2^{n \Delta_{n}}$, which then becomes a constant multiplicative factor of the upper bound. Since both inequalities hold for every $\theta$, whereas $\bar{P}_{e, u}(R, n)$ is independent of $\theta$, we have actually proved the inequalities

$$
\bar{P}_{e, u}(R, n) \leq 2 \cdot \min _{\theta \in \Theta} \bar{P}_{e, \theta}\left(R+\Delta_{n}, n\right)
$$

and

$$
\bar{P}_{e, u}(R, n) \leq 2 \cdot 2^{n \Delta_{n}} \cdot \min _{\theta \in \Theta} \bar{P}_{e, \theta}(R, n) .
$$

This completes the proof of Theorem 1.

One of the elegant points in [21] is that the universality of the proposed decoding metric, in the random coding error exponent sense, is proved using a comparative analysis, without recourse to an explicit derivation of the random coding error exponent of the optimum decoder. The above proof of Theorem 1 has the same feature. However, thanks to Shulman's lower bound on the probability of a union of events, the proof here is both simpler and more general than in [21], in several respects: (i) it allows a general random coding distribution $Q$, not just the uniform distribution, (ii) it requires only pairwise independence and not full independence between the codewords, and (iii) it assumes nothing concerning the underlying channel. Indeed, it will be seen shortly How Ziv's universal decoding metric is obtained as a special case of our approach.

We summarize a few important points:

1. We have defined a fairly general framework for universal decoding, allowing a general random coding distribution $Q$, a general channel, and a a general family of decoding metrics $\left\{m_{\theta}, \theta \in\right.$ $\Theta$ \}. Most of the previous works in universal decoding, mentioned in the second and the third 
paragraphs of the Introduction, relate to the special case where the ML decoder for the given channel is equivalent to $m_{\theta}$ for a certain choice of $\theta$.

2. Another special case that falls within our framework is mismatched decoding: In this case, $\Theta$ is a singleton and the unique decoding metric $m_{\theta}$ in this singleton is different from the ML decoding metric of the actual channel.

3. Yet another special case is the case where the channel is deterministic. This is partially related to the "individual channel" paradigm due to Lomnitz and Feder (see, e.g., [11], [12] among many other papers), Misra and Weissman [16], and Shayevitz and Feder [18]. The main difference is that here, we are not concerned with universality of the encoder, as we simply assume a fixed random coding distribution. In the absence of feedback, there is no hope for universal encoding.

\section{Useful Approximations of the Universal Decoding Metric}

In some situations, it may not be a trivial task to evaluate $Q[\mathcal{T}(\boldsymbol{x} \mid \boldsymbol{y})]$, which is needed in order to implement the proposed universal decoding metric. Suppose, however, that one can uniformly lower bound $Q[\mathcal{T}(\boldsymbol{x} \mid \boldsymbol{y})]=\exp \{-n U(\boldsymbol{x}, \boldsymbol{y})\}$ by $\exp \left\{-n U^{\prime}(\boldsymbol{x}, \boldsymbol{y})\right\}$, for some function $U^{\prime}(\boldsymbol{x}, \boldsymbol{y})$ which is computable and suppose that $U^{\prime}(\cdot, \cdot)$ is not too large in the sense that it satisfies the following condition:

$$
\max _{\boldsymbol{y} \in \mathcal{Y}^{n}} \sum_{\boldsymbol{x} \in \mathcal{X}^{n}} Q(\boldsymbol{x}) 2^{n U^{\prime}(\boldsymbol{x}, \boldsymbol{y})} \leq 2^{n \Delta_{n}^{\prime}}
$$

where $\Delta_{n}^{\prime} \rightarrow 0$. We argue that in such a case, $U^{\prime}(\cdot, \cdot)$ can replace $U(\cdot, \cdot)$ as a universal decoding metric and Theorem 1 remains valid.

To see why this is true, first observe that $\bar{\Pi}_{e, \theta}(\boldsymbol{x}, \boldsymbol{y})$ is trivially lower bounded by $\exp \left\{-n U^{\prime}(\boldsymbol{x}, \boldsymbol{y})\right\}$, following (34) and the very definition of $U^{\prime}(\boldsymbol{x}, \boldsymbol{y})$ as an upper bound on $U(\boldsymbol{x}, \boldsymbol{y})$. As for the upper bound, we have

$$
\begin{aligned}
\bar{\Pi}_{e, u^{\prime}}(\boldsymbol{x}, \boldsymbol{y}) & \triangleq \sum_{\left\{\boldsymbol{x}^{\prime}: U^{\prime}\left(\boldsymbol{x}^{\prime}, \boldsymbol{y}\right) \geq U^{\prime}(\boldsymbol{x}, \boldsymbol{y})\right\}} Q\left(\boldsymbol{x}^{\prime}\right) \\
& =\exp \left[-n U^{\prime}(\boldsymbol{x}, \boldsymbol{y})\right] \cdot \sum_{\left\{\boldsymbol{x}^{\prime}: U^{\prime}\left(\boldsymbol{x}^{\prime}, \boldsymbol{y}\right) \geq U^{\prime}(\boldsymbol{x}, \boldsymbol{y})\right\}} Q\left(\boldsymbol{x}^{\prime}\right) \exp \left[n U^{\prime}(\boldsymbol{x}, \boldsymbol{y})\right]
\end{aligned}
$$




$$
\begin{aligned}
& \leq \exp \left[-n U^{\prime}(\boldsymbol{x}, \boldsymbol{y})\right] \cdot \sum_{\left\{\boldsymbol{x}^{\prime}: U^{\prime}\left(\boldsymbol{x}^{\prime}, \boldsymbol{y}\right) \geq U^{\prime}(\boldsymbol{x}, \boldsymbol{y})\right\}} Q\left(\boldsymbol{x}^{\prime}\right) \exp \left[n U^{\prime}\left(\boldsymbol{x}^{\prime}, \boldsymbol{y}\right)\right] \\
& \leq \exp \left[-n U^{\prime}(\boldsymbol{x}, \boldsymbol{y})\right] \cdot \sum_{\boldsymbol{x}^{\prime} \in \mathcal{X}^{n}} Q\left(\boldsymbol{x}^{\prime}\right) \exp \left[n U^{\prime}\left(\boldsymbol{x}^{\prime}, \boldsymbol{y}\right)\right] \\
& \leq \exp \left\{-n\left[U^{\prime}(\boldsymbol{x}, \boldsymbol{y})-\Delta_{n}^{\prime}\right]\right\} .
\end{aligned}
$$

Now, the corresponding upper bounds on $\bar{P}_{e, u^{\prime}}(R, n)$, in terms of $\min _{\theta} P_{e, \theta}(R, n)$, are derived as before, just with $U$ replaced by $U^{\prime}$. The price of passing from $U$ to $U^{\prime}$ might be in a slowdown of the convergence of $\Delta_{n}^{\prime}$ vs. $\Delta_{n}$. For example, $U^{\prime}$ might correspond to more refined equivalence classes $\{\mathcal{T}(\boldsymbol{x} \mid \boldsymbol{y})\}$.

As an example of the usefulness of this result, let us refer to Ziv's universal decoding metric for finite-state channels [21]. In particular, let $\mathcal{M}$ be the class of decoding metrics corresponding to finite-state channels, defined as follows: For a given $\boldsymbol{x} \in \mathcal{X}^{n}$ and $\boldsymbol{y} \in \mathcal{Y}^{n}$, let $\boldsymbol{s}=\left(s_{1}, \ldots, s_{n}\right) \in \mathcal{S}^{n}$ $(\mathcal{S}$ being a finite set), be a sequence generated recursively according to

$$
s_{i+1}=g\left(x_{i}, y_{i}, s_{i}\right), \quad i=1, \ldots, n-1,
$$

where $s_{1}$ is some fixed initial state and $g: \mathcal{X} \times \mathcal{Y} \times \mathcal{S} \rightarrow \mathcal{S}$ is a certain next-state function. Now define

$$
m_{\theta}(\boldsymbol{x}, \boldsymbol{y})=\sum_{i=1}^{n} \theta\left(x_{i}, y_{i}, s_{i}\right)
$$

where $\{\theta(x, y, s), x \in \mathcal{X}, y \in \mathcal{Y}, s \in \mathcal{S}\}$ are arbitrary real valued parameters. Similarly as in [21], suppose that $Q(\boldsymbol{x})$ is the uniform distribution over $\mathcal{X}^{n}$. Then $Q[\mathcal{T}(\boldsymbol{x} \mid \boldsymbol{y})]$ is proportional to $|\mathcal{T}(\boldsymbol{x} \mid \boldsymbol{y})|$, but the problem is that here, unlike in Example 1, there is no apparent single-letter expression $^{8}$ for the exponential growth rate of $|\mathcal{T}(\boldsymbol{x} \mid \boldsymbol{y})|$ in general (unless the state variable in eq. (43) depends solely on the previous state and the previous channel output). Moreover, $|\mathcal{T}(\boldsymbol{x} \mid \boldsymbol{y})|$ depends on the next-state function $g$ in eq. (43), which is assumed unknown. Fortunately enough, however, $|\mathcal{T}(\boldsymbol{x} \mid \boldsymbol{y})|$, in this case, can be lower bounded [21, Lemma 1] by

$$
|\mathcal{T}(\boldsymbol{x} \mid \boldsymbol{y})| \geq 2^{L Z(\boldsymbol{x} \mid \boldsymbol{y})-n o(n)}
$$

where $L Z(\boldsymbol{x} \mid \boldsymbol{y})$ denotes the length (in bits) of the conditional Lempel-Ziv code (see [21, proof of Lemma 2], [14]) of $\boldsymbol{x}$ when $\boldsymbol{y}$ is given as side information at both encoder and decoder. Consequently,

\footnotetext{
${ }^{8}$ In a nutshell, had there been such a single-letter expression, one could have easily derived a single-letter expression for the entropy rate of a hidden Markov process [1, Section 4.5] using the method of types.
} 
one can upper bound $U(\boldsymbol{x}, \boldsymbol{y})$ by

$$
U^{\prime}(\boldsymbol{x}, \boldsymbol{y})=\log |\mathcal{X}|-\frac{L Z(\boldsymbol{x} \mid \boldsymbol{y})}{n}+o(n)
$$

as our decoding metric. Indeed, eq. (37) is satisfied by this choice of $U^{\prime}$ since

$$
\begin{aligned}
\sum_{\boldsymbol{x}} Q(\boldsymbol{x}) 2^{n U^{\prime}(\boldsymbol{x}, \boldsymbol{y})} & =\sum_{\boldsymbol{x}} \frac{2^{n U^{\prime}(\boldsymbol{x}, \boldsymbol{y})}}{|\mathcal{X}|^{n}} \\
& =\sum_{\boldsymbol{x}} 2^{-L Z(\boldsymbol{x} \mid \boldsymbol{y})+n o(n)} \\
& \leq 2^{n o(n)},
\end{aligned}
$$

where the last equality is Kraft's inequality which holds since $L Z(\boldsymbol{x} \mid \boldsymbol{y})$ is a length function of $\boldsymbol{x}$ for every $\boldsymbol{y}$. This explains why Ziv's decoder, which selects the message $i$ with the minimum of $L Z\left(\boldsymbol{x}_{i} \mid \boldsymbol{y}\right)$, is universally asymptotically optimum in the random coding exponent sense. Note that the assumption that $Q$ is uniform is not really essential here. In fact, $Q$ can also be any exchangeable probability distribution (i.e., $\boldsymbol{x}^{\prime}$ is a permutation of $\boldsymbol{x}$ implies $\left.Q\left(\boldsymbol{x}^{\prime}\right)=Q(\boldsymbol{x})\right)$. Moreover, if the state variable $s_{i}$ includes a component, say, $\sigma_{i}$, that is fed merely by $\left\{x_{i}\right\}$ (but not $\left\{y_{i}\right\}$ ), then it is enough that $Q$ would be invariant within conditional types of $\boldsymbol{x}$ given $\boldsymbol{\sigma}=\left(\sigma_{1}, \ldots, \sigma_{n}\right)$. In such a case, we would have

$$
U^{\prime}(\boldsymbol{x} \cdot \boldsymbol{y})=-\frac{1}{n}[\log Q(\boldsymbol{x})+L Z(\boldsymbol{x} \mid \boldsymbol{y})]
$$

\section{Extensions}

We now demonstrate how our method extends to more involved scenarios of communication systems. The first extension corresponds to random coding distributions that allow access to noiseless feedback. While this extension is not complicated, it is important from the operational point of view, because feedback allows the encoder to learn the channel and thereby to adapt the random coding distribution to the channel statistical characteristics.

Our second extension is to the problem of universal decoding for multiple access channels (MAC's) with respect to a given class of decoding metrics (again, without feedback, but the extension that combines feedback is again straightforward). This extension is deliberately not provided in full generality in the sense that we make a certain facilitating assumption on the structure of the class of decoding metrics, in order to make the analysis simpler. The main point here is not 
the quest for full generality, but to demonstrate that this extension, even under this facilitating assumption, is not a trivial task since the universal decoding metric has to confront three different types of error events (in the case of a MAC with two senders): (i) the event were both messages are decoded incorrectly, (ii) the event where only the message of sender no. 1 is decoded incorrectly, and (iii) the event where only the message of sender no. 2 is decoded incorrectly. As a consequence, it turns out that the resulting universal decoding metric is surprisingly different from those of earlier works on universal decoding for the MAC [10], [5, Section VIII], [17], mostly because the problem setting here is different (and more general) from those of these earlier works (in the sense that the universality here is relative to a given class of decoders while the underlying channel is arbitrary, and not relative to a given class of channels). While we are not arguing that all the universal decoders of these previous articles are necessarily suboptimum in our scenario, we are able to prove the universality only for our own universal decoding metric.

\subsection{Feedback}

In the paradigm of random coding in the presence of feedback, it is convenient to think of an independent random selection of symbols of $\mathcal{X}$ along a tree whose branches are labeled by

$$
\left\{y_{1}\right\},\left\{y_{1}, y_{2}\right\}, \ldots,\left\{y_{1}, \ldots, y_{n-1}\right\}
$$

for all possible outcomes of these vectors. Accordingly, the random coding distribution $Q(\boldsymbol{x})$ is replaced by

$$
Q(\boldsymbol{x} \mid \boldsymbol{y}) \triangleq \prod_{i=1}^{n} Q\left(x_{i} \mid x^{i-1}, y^{i-1}\right) .
$$

Thus, each message $i \in\{1,2, \ldots, M\}$ is represented by a complete tree of depth $n$ and $|\mathcal{Y}|^{n-1}$ leaves. Theorem 1 and its proof remain intact with $Q(\cdot)$ being replaced by $Q(\cdot \mid \boldsymbol{y})$ in all places. Thus, the universal decoding metric is redefined as

$$
U(\boldsymbol{x}, \boldsymbol{y})=-\frac{1}{n} \log Q[\mathcal{T}(\boldsymbol{x} \mid \boldsymbol{y}) \mid \boldsymbol{y}],
$$

the expectation in eqs. (32) and (34) is redefined w.r.t.

$$
P(\boldsymbol{x}, \boldsymbol{y})=\prod_{i=1}^{n}\left[Q\left(x_{i} \mid x^{i-1}, y^{i-1}\right) P\left(y_{i} \mid x^{i}, y^{i-1}\right)\right],
$$

and in condition (37), $Q(\boldsymbol{x})$ is replaced by $Q(\boldsymbol{x} \mid \boldsymbol{y})$. 
One might limit the structure of the feedback, for example, by letting each $Q\left(\cdot \mid x^{i-1}, y^{i-1}\right)$ depend on $\left(x^{i-1}, y^{i-1}\right)$ only via a state variable $t_{i}$ fed by these two sequences, i.e.,

$$
t_{i}=g\left(t_{i-1}, x_{i-1}, y_{i-1}\right)
$$

that is

$$
Q(\boldsymbol{x} \mid \boldsymbol{y})=\prod_{i=1}^{n} Q\left(x_{i} \mid x^{i-1}, y^{i-1}\right)=\prod_{i=1}^{n} Q\left(x_{i} \mid t_{i}\right) .
$$

In the above example of decoding metrics corresponding to finite-state channels, one can refine the equivalence classes to include the information about $t_{i}$ (see Section 4), and then $Q$ would be invariant within a type class $T_{\boldsymbol{x} \mid \boldsymbol{y}, \boldsymbol{s}, \boldsymbol{t}}$, where $\boldsymbol{t}=\left(t_{1}, \ldots, t_{n}\right)$. In this case, the decoding metric $U^{\prime}$ would become

$$
U^{\prime}(\boldsymbol{x}, \boldsymbol{y})=-\frac{1}{n}[\log Q(\boldsymbol{x} \mid \boldsymbol{y})+L Z(\boldsymbol{x} \mid \boldsymbol{y})],
$$

where $Q(\boldsymbol{x} \mid \boldsymbol{y})$ is understood to be defined according to eq. (55).

\subsection{The Multiple Access Channel}

Consider an arbitrary multiple access channel (MAC), namely, a channel with two inputs, $\boldsymbol{x}_{1}$ and $\boldsymbol{x}_{2}$, and one output $\boldsymbol{y}$. The two inputs are used by two different users which do not cooperate. User no. 1 generates $M_{1}=2^{n R_{1}}$ independent codewords, $\boldsymbol{x}_{1}(1), \ldots, \boldsymbol{x}_{1}\left(M_{1}\right)$, using a random coding distribution $Q_{1}$, and user no. 2 generates $M_{2}=2^{n R_{2}}$ independent codewords, $\boldsymbol{x}_{2}(1), \ldots, \boldsymbol{x}_{2}\left(M_{2}\right)$, using a random coding distribution $Q_{2} \cdot{ }^{9}$

We define a class $\mathcal{M}$ of decoding metrics $\left\{m_{\theta}\left(\boldsymbol{x}_{1}, \boldsymbol{x}_{2}, \boldsymbol{y}\right), \theta \in \Theta\right\}$. Decoder $\mathcal{D}_{\theta}$ picks the pair of messages $\left(\boldsymbol{x}_{1}(i), \boldsymbol{x}_{2}(j)\right), i \in\left\{1, \ldots, M_{1}\right\}, j \in\left\{1, \ldots, M_{2}\right\}$, which maximizes $m_{\theta}\left(\boldsymbol{x}_{1}(i), \boldsymbol{x}_{2}(j), \boldsymbol{y}\right)$.

We assume that the random coding ensemble and the class of decoders is such that for every $\boldsymbol{y}, m_{\theta}\left(\boldsymbol{X}_{1}(i), \boldsymbol{X}_{2}(j), \boldsymbol{y}\right)$ and $m_{\theta}\left(\boldsymbol{X}_{1}\left(i^{\prime}\right), \boldsymbol{X}_{2}\left(j^{\prime}\right), \boldsymbol{y}\right)$ are statistically independent whenever $(i, j) \neq$ $\left(i^{\prime}, j^{\prime}\right)$. While this requirement is easily satisfied when the both $i \neq i^{\prime}$ and $j \neq j^{\prime}$ (for example, when all codewords are drawn by independent random selection), it is less obvious for combinations of pairs $(i, j)$ and $\left(i^{\prime}, j^{\prime}\right)$ for which either $i=i^{\prime}$ or $j=j^{\prime}$ (but, of course, not both). Still, this requirement is satisfied, for example, if $\mathcal{X}_{1}=\mathcal{X}_{2}=\{0,1, \ldots, K-1\}$ (or the continuous interval

\footnotetext{
${ }^{9}$ We should point out that a more general model definition should allow time-sharing, which means that the codewords of both users should be drawn conditionally independently given a sequence $s$ the designates the timesharing protocol known to all parties. This will just amount to conditioning many quantities on $\boldsymbol{s}$. For the sake of simplicity of the exposition, we will not add this conditioning on $\boldsymbol{s}$.
} 
$[0, A]), Q_{1}$ and $Q_{2}$ are both uniform across the alphabet, and $m_{\theta}\left(\boldsymbol{x}_{1}, \boldsymbol{x}_{2}, \boldsymbol{y}\right)$ depends on $\boldsymbol{x}_{1}$ and $\boldsymbol{x}_{2}$ only via $\boldsymbol{x}_{1} \oplus \boldsymbol{x}_{2}$, where $\oplus$ denotes addition modulo $K$ (or addition modulo $A$, in the example of the continuous case). Decoding metrics with this property are motivated by classes of multiple access channels, $P\left(\boldsymbol{y} \mid \boldsymbol{x}_{1}, \boldsymbol{x}_{2}\right)$, in which the users interfere with each other additively, i.e., $P\left(\boldsymbol{y} \mid \boldsymbol{x}_{1}, \boldsymbol{x}_{2}\right)=$ $W\left(\boldsymbol{y} \mid \boldsymbol{x}_{1} \oplus \boldsymbol{x}_{2}\right)$. Still, the dependence of $\boldsymbol{y}$ on $\boldsymbol{x}_{1} \oplus \boldsymbol{x}_{2}$ can be arbitrary. In other words, the channel is known to depend only on the modulo 2 sum of the inputs, but the form of this dependence may not be known. Another example where the above independence requirement is met is when $\mathcal{X}_{1}=\mathcal{X}_{2}=\{-1,+1\}$ and $m_{\theta}$ depends on $\boldsymbol{x}_{1}$ and $\boldsymbol{x}_{2}$ only via their component-wise product $\boldsymbol{x}_{1} \cdot \boldsymbol{x}_{2}$.

We now define three kinds of equivalence classes:

$$
\begin{aligned}
\mathcal{T}\left(\boldsymbol{x}_{1}, \boldsymbol{x}_{2} \mid \boldsymbol{y}\right) & =\left\{\left(\boldsymbol{x}_{1}^{\prime}, \boldsymbol{x}_{2}^{\prime}\right): \forall \theta \in \Theta m_{\theta}\left(\boldsymbol{x}_{1}^{\prime}, \boldsymbol{x}_{2}^{\prime}, \boldsymbol{y}\right)=m_{\theta}\left(\boldsymbol{x}_{1}, \boldsymbol{x}_{2}, \boldsymbol{y}\right)\right\} \\
\mathcal{T}\left(\boldsymbol{x}_{1} \mid \boldsymbol{x}_{2}, \boldsymbol{y}\right) & =\left\{\boldsymbol{x}_{1}^{\prime}: \forall \theta \in \Theta m_{\theta}\left(\boldsymbol{x}_{1}^{\prime}, \boldsymbol{x}_{2}, \boldsymbol{y}\right)=m_{\theta}\left(\boldsymbol{x}_{1}, \boldsymbol{x}_{2}, \boldsymbol{y}\right)\right\} \\
& =\left\{\boldsymbol{x}_{1}^{\prime}:\left(\boldsymbol{x}_{1}^{\prime}, \boldsymbol{x}_{2}\right) \in \mathcal{T}\left(\boldsymbol{x}_{1}, \boldsymbol{x}_{2} \mid \boldsymbol{y}\right)\right\} \\
\mathcal{T}\left(\boldsymbol{x}_{2} \mid \boldsymbol{x}_{1}, \boldsymbol{y}\right) & =\left\{\boldsymbol{x}_{2}^{\prime}: \forall \theta \in \Theta m_{\theta}\left(\boldsymbol{x}_{1}, \boldsymbol{x}_{2}^{\prime}, \boldsymbol{y}\right)=m_{\theta}\left(\boldsymbol{x}_{1}, \boldsymbol{x}_{2}, \boldsymbol{y}\right)\right\} \\
& =\left\{\boldsymbol{x}_{2}^{\prime}:\left(\boldsymbol{x}_{1}, \boldsymbol{x}_{2}^{\prime}\right) \in \mathcal{T}\left(\boldsymbol{x}_{1}, \boldsymbol{x}_{2} \mid \boldsymbol{y}\right)\right\}
\end{aligned}
$$

We also assume, as before, that for every $\boldsymbol{y}$, the number of different type classes $\left\{\mathcal{T}\left(\boldsymbol{x}_{1}, \boldsymbol{x}_{2} \mid \boldsymbol{y}\right)\right\}$ is upper bounded by $2^{n \Delta_{n}}$. Next, define the following functions:

$$
\begin{aligned}
U_{0}\left(\boldsymbol{x}_{1}, \boldsymbol{x}_{2}, \boldsymbol{y}\right) & =-\frac{1}{n} \log \left\{\left(Q_{1} \times Q_{2}\right)\left[\mathcal{T}\left(\boldsymbol{x}_{1}, \boldsymbol{x}_{2} \mid \boldsymbol{y}\right)\right]\right\} \\
U_{1}\left(\boldsymbol{x}_{1}, \boldsymbol{x}_{2}, \boldsymbol{y}\right) & =-\frac{1}{n} \log Q_{1}\left[\mathcal{T}\left(\boldsymbol{x}_{1} \mid \boldsymbol{x}_{2}, \boldsymbol{y}\right)\right] \\
U_{2}\left(\boldsymbol{x}_{1}, \boldsymbol{x}_{2}, \boldsymbol{y}\right) & =-\frac{1}{n} \log Q_{2}\left[\mathcal{T}\left(\boldsymbol{x}_{2} \mid \boldsymbol{x}_{1}, \boldsymbol{y}\right)\right] .
\end{aligned}
$$

What makes the MAC interesting, in the context of universal decoding, is that the universal decoder has to cope with three different types of errors: (i) both messages are decoded incorrectly, (ii) the message of user no. 2 is decoded correctly, but that of user no. 1 is not, and (iii) like (ii), but with the roles of the users swapped. From Theorem 1 and its proof (after an obvious modification), it is apparent that had only errors of type (i) existed, then $U_{0}$ could have been a universal decoding metric. Similarly, had only errors of type (ii) existed, then $U_{1}$ could be a universal decoding metric, and by the same token, for error of type (iii) alone, one would use $U_{2}$. However, in reality, all three types of error events might occur and we need one universal decoding metric that handles all of 
them at the same time. The question is then how to combine $U_{0}, U_{1}$ and $U_{2}$ into one metric that would work at least as well as the best decoder in the given class.

The answer turns out to be the following: Define the universal decoding metric as

$$
U\left(\boldsymbol{x}_{1}, \boldsymbol{x}_{2}, \boldsymbol{y}\right)=\min \left\{\left[U_{0}\left(\boldsymbol{x}_{1}, \boldsymbol{x}_{2}, \boldsymbol{y}\right)-R_{1}-R_{2}\right],\left[U_{1}\left(\boldsymbol{x}_{1}, \boldsymbol{x}_{2}, \boldsymbol{y}\right)-R_{1}\right],\left[U_{2}\left(\boldsymbol{x}_{1}, \boldsymbol{x}_{2}, \boldsymbol{y}\right)-R_{2}\right]\right\}
$$

We argue that $U\left(\boldsymbol{x}_{1}, \boldsymbol{x}_{2}, \boldsymbol{y}\right)$ competes favorably with the best $m_{\theta}$ in a sense analogous to that asserted in Theorem 1. This decoding metric is different from the universal decoding metrics used for the MAC, for example, in [17] and [10], which were based on the MMI decoder and the minimum empirical conditional entropy (minimum equivocation) rule, respectively. It is not argued here that these decoding rules are necessarily suboptimal in the present setting, but on the other hand, we do not have a proof that they compete favorably with the best decoder in the class $\mathcal{M}$. The remaining part of this section is devoted to a description of the main modifications and extensions needed in the proof of Theorem 1 in order to prove the universality of $U\left(\boldsymbol{x}_{1}, \boldsymbol{x}_{2}, \boldsymbol{y}\right)$ for the MAC.

The pairwise probability of type (i) error for an arbitrary decoder in the reference class $\mathcal{M}$ is lower bounded by

$$
\begin{aligned}
P_{e, \theta}^{(i)}\left(\boldsymbol{x}_{1}, \boldsymbol{x}_{2}, \boldsymbol{y}\right) & \triangleq \sum_{\left\{\boldsymbol{x}_{1}^{\prime}, \boldsymbol{x}_{2}^{\prime}: m_{\theta}\left(\boldsymbol{x}_{1}^{\prime}, \boldsymbol{x}_{2}^{\prime}, \boldsymbol{y}\right) \geq m_{\theta}\left(\boldsymbol{x}_{1}, \boldsymbol{x}_{2}, \boldsymbol{y}\right)\right\}} Q_{1}\left(\boldsymbol{x}_{1}^{\prime}\right) Q_{2}\left(\boldsymbol{x}_{2}^{\prime}\right) \\
& \geq \sum_{\left(\boldsymbol{x}_{1}^{\prime}, \boldsymbol{x}_{2}^{\prime}\right) \in \mathcal{T}\left(\boldsymbol{x}_{1}, \boldsymbol{x}_{2} \mid \boldsymbol{y}\right)} Q_{1}\left(\boldsymbol{x}_{1}^{\prime}\right) Q_{2}\left(\boldsymbol{x}_{2}^{\prime}\right) \\
& =\left(Q_{1} \times Q_{2}\right)\left[\mathcal{T}\left(\boldsymbol{x}_{1}, \boldsymbol{x}_{2} \mid \boldsymbol{y}\right)\right] \\
& =2^{-n U_{0}\left(\boldsymbol{x}_{1}, \boldsymbol{x}_{2}, \boldsymbol{y}\right)}
\end{aligned}
$$

As for the pairwise error probability of type (ii), we have

$$
\begin{aligned}
P_{e, \theta}^{(i i)}\left(\boldsymbol{x}_{1}, \boldsymbol{x}_{2}, \boldsymbol{y}\right) & \triangleq \sum_{\left\{\boldsymbol{x}_{1}^{\prime}: m_{\theta}\left(\boldsymbol{x}_{1}^{\prime}, \boldsymbol{x}_{2}, \boldsymbol{y}\right) \geq m_{\theta}\left(\boldsymbol{x}_{1}, \boldsymbol{x}_{2}, \boldsymbol{y}\right)\right\}} Q_{1}\left(\boldsymbol{x}_{1}^{\prime}\right) \\
& \geq \sum_{\boldsymbol{x}_{1}^{\prime} \in \mathcal{T}\left(\boldsymbol{x}_{1} \mid \boldsymbol{x}_{2}, \boldsymbol{y}\right)} Q_{1}\left(\boldsymbol{x}_{1}^{\prime}\right) \\
& =Q_{1}\left[\mathcal{T}\left(\boldsymbol{x}_{1} \mid \boldsymbol{x}_{2}, \boldsymbol{y}\right)\right] \\
& =2^{-n U_{1}\left(\boldsymbol{x}_{1}, \boldsymbol{x}_{2}, \boldsymbol{y}\right)}
\end{aligned}
$$

and similarly, for type (iii):

$$
P_{e, \theta}^{(i i i)}\left(\boldsymbol{x}_{1}, \boldsymbol{x}_{2}, \boldsymbol{y}\right) \geq 2^{-n U_{2}\left(\boldsymbol{x}_{1}, \boldsymbol{x}_{2}, \boldsymbol{y}\right)}
$$


Let $\mathcal{I}$ be half of the set $\left\{1,2, \ldots, M_{1}\right\}-\{i\}$ and let $\mathcal{J}$ be half of the set $\left\{1,2, \ldots, M_{2}\right\}-\{j\}(i$ and $j$ being the correct messages of the two senders). Let $\mathcal{A}$ be the set of all $\left(M_{1}-1\right)\left(M_{2}-1\right) / 4$ pairs $\left(i^{\prime}, j^{\prime}\right) \in \mathcal{I}^{c} \times \mathcal{J}^{c}$, where both $i^{\prime} \neq i$ and $j^{\prime} \neq j$. Under our above assumptions, the following is true: given $\left(\boldsymbol{x}_{1}(i), \boldsymbol{x}_{2}(j), \boldsymbol{y}\right)$, the events

$$
\begin{gathered}
\left\{m_{\theta}\left(\boldsymbol{X}_{1}\left(i^{\prime}\right), \boldsymbol{x}_{2}(j), \boldsymbol{y}\right) \geq m_{\theta}\left(\boldsymbol{x}_{1}(i), \boldsymbol{x}_{2}(j), \boldsymbol{y}\right)\right\}_{i^{\prime} \in \mathcal{I}}, \\
\left\{m_{\theta}\left(\boldsymbol{x}_{1}(i), \boldsymbol{X}_{2}\left(j^{\prime}\right), \boldsymbol{y}\right) \geq m_{\theta}\left(\boldsymbol{x}_{1}(i), \boldsymbol{x}_{2}(j), \boldsymbol{y}\right)\right\}_{j^{\prime} \in \mathcal{J}},
\end{gathered}
$$

and

$$
\left\{m_{\theta}\left(\boldsymbol{X}_{1}\left(i^{\prime}\right), \boldsymbol{X}_{2}\left(j^{\prime}\right), \boldsymbol{y}\right) \geq m_{\theta}\left(\boldsymbol{x}_{1}(i), \boldsymbol{x}_{2}(j), \boldsymbol{y}\right)\right\}_{\left(i^{\prime}, j^{\prime}\right) \in \mathcal{A}}
$$

are all pairwise independent. Defining the set of pairs $\mathcal{B}=\mathcal{A} \cup[\{i\} \times \mathcal{J}] \cup[\mathcal{I} \times\{j\}]$, the total probability of error, associated with the decoder $\mathcal{D}_{\theta}$, is lower bounded as follows:

$$
\begin{aligned}
& \bar{P}_{e, \theta}\left(R_{1}, R_{2}, n\right) \\
\triangleq & \frac{1}{M_{1} M_{2}} \sum_{i=1}^{M_{1}} \sum_{j=1}^{M_{2}} \operatorname{Pr} \bigcup_{\left(i^{\prime}, j^{\prime}\right) \neq(i, j)}\left\{m_{\theta}\left(\boldsymbol{X}_{1}\left(i^{\prime}\right), \boldsymbol{X}_{2}\left(j^{\prime}\right), \boldsymbol{Y}\right) \geq m_{\theta}\left(\boldsymbol{X}_{1}(i), \boldsymbol{X}_{2}(j), \boldsymbol{Y}\right) \mid(i, j) \text { sent }\right\}(74) \\
\geq & \frac{1}{M_{1} M_{2}} \sum_{i=1}^{M_{1}} \sum_{j=1}^{M_{2}} \operatorname{Pr} \bigcup_{\left(i^{\prime}, j^{\prime}\right) \in \mathcal{B}}\left\{m_{\theta}\left(\boldsymbol{X}_{1}\left(i^{\prime}\right), \boldsymbol{X}_{2}\left(j^{\prime}\right), \boldsymbol{Y}\right) \geq m_{\theta}\left(\boldsymbol{X}_{1}(i), \boldsymbol{X}_{2}(j), \boldsymbol{Y}\right) \mid(i, j) \text { sent }\right\} \\
\geq & \boldsymbol{E} \min \left\{1, \frac{\left(M_{1}-1\right)\left(M_{2}-1\right)}{4} \cdot 2^{-n U_{0}\left(\boldsymbol{X}_{1}, \boldsymbol{X}_{2}, \boldsymbol{Y}\right)}+\right. \\
& \left.\frac{\left(M_{1}-1\right)}{2} \cdot 2^{-n U_{1}\left(\boldsymbol{X}_{1}, \boldsymbol{X}_{2}, \boldsymbol{Y}\right)}+\frac{\left(M_{2}-1\right)}{2} \cdot 2^{-n U_{2}\left(\boldsymbol{X}_{1}, \boldsymbol{X}_{2}, \boldsymbol{Y}\right)}\right\} \\
\doteq & \boldsymbol{E} \min \left\{1,2^{-n\left[U_{0}\left(\boldsymbol{X}_{1}, \boldsymbol{X}_{2}, \boldsymbol{Y}\right)-R_{1}-R_{2}\right]}+\right. \\
& \left.2^{-n\left[U_{1}\left(\boldsymbol{X}_{1}, \boldsymbol{X}_{2}, \boldsymbol{Y}\right)-R_{1}\right]}+2^{-n\left[U_{2}\left(\boldsymbol{X}_{1}, \boldsymbol{X}_{2}, \boldsymbol{Y}\right)-R_{2}\right]}\right\} \\
\doteq & \boldsymbol{E} \min \left\{1,2^{-n U\left(\boldsymbol{X}_{1}, \boldsymbol{X}_{2}, \boldsymbol{Y}\right)}\right\} \\
= & \boldsymbol{E}\left\{2^{-n\left[U\left(\boldsymbol{X}_{1}, \boldsymbol{X}_{2}, \boldsymbol{Y}\right)\right]_{+}}\right\},
\end{aligned}
$$

where the second inequality is again due to Shulman [19, Lemma A.2]. Consider now the function $U\left(\boldsymbol{x}_{1}, \boldsymbol{x}_{2}, \boldsymbol{y}\right)$ as a universal decoding metric. Then, we have the following:

$$
\begin{aligned}
P_{e, u}^{(i)}\left(\boldsymbol{x}_{1}, \boldsymbol{x}_{2}, \boldsymbol{y}\right) & \triangleq \sum_{\left\{\left(\boldsymbol{x}_{1}^{\prime}, \boldsymbol{x}_{2}^{\prime}\right): U\left(\boldsymbol{x}_{1}^{\prime}, \boldsymbol{x}_{2}^{\prime}, \boldsymbol{y}\right) \geq U\left(\boldsymbol{x}_{1}, \boldsymbol{x}_{2}, \boldsymbol{y}\right)\right\}} Q_{1}\left(\boldsymbol{x}_{1}^{\prime}\right) Q_{2}\left(\boldsymbol{x}_{2}^{\prime}\right) \\
& =\sum_{\left\{\mathcal{T}\left(\boldsymbol{x}_{1}^{\prime}, \boldsymbol{x}_{2}^{\prime} \mid \boldsymbol{y}\right): U\left(\boldsymbol{x}_{1}^{\prime}, \boldsymbol{x}_{2}^{\prime}, \boldsymbol{y}\right) \geq U\left(\boldsymbol{x}_{1}, \boldsymbol{x}_{2}, \boldsymbol{y}\right)\right\}} \sum_{\left(\tilde{\boldsymbol{x}}_{1}, \tilde{\boldsymbol{x}}_{2}\right) \in \mathcal{T}\left(\boldsymbol{x}_{1}^{\prime}, \boldsymbol{x}_{2}^{\prime} \mid \boldsymbol{y}\right)} Q_{1}\left(\tilde{\boldsymbol{x}}_{1}\right) Q_{2}\left(\tilde{\boldsymbol{x}}_{2}\right)
\end{aligned}
$$




$$
\begin{aligned}
& \sum_{\left\{\mathcal{T}\left(\boldsymbol{x}_{1}^{\prime}, \boldsymbol{x}_{2}^{\prime} \mid \boldsymbol{y}\right): U\left(\boldsymbol{x}_{1}^{\prime}, \boldsymbol{x}_{2}^{\prime}, \boldsymbol{y}\right) \geq U\left(\boldsymbol{x}_{1}, \boldsymbol{x}_{2}, \boldsymbol{y}\right)\right\}}\left(Q_{1} \times Q_{2}\right)\left[\mathcal{T}\left(\boldsymbol{x}_{1}^{\prime}, \boldsymbol{x}_{2}^{\prime} \mid \boldsymbol{y}\right)\right] \\
& \sum_{\left\{\mathcal{T}\left(\boldsymbol{x}_{1}^{\prime}, \boldsymbol{x}_{2}^{\prime} \mid \boldsymbol{y}\right): U\left(\boldsymbol{x}_{1}^{\prime}, \boldsymbol{x}_{2}^{\prime}, \boldsymbol{y}\right) \geq U\left(\boldsymbol{x}_{1}, \boldsymbol{x}_{2}, \boldsymbol{y}\right)\right\}} \exp \left[-n U_{0}\left(\boldsymbol{x}_{1}^{\prime}, \boldsymbol{x}_{2}^{\prime}, \boldsymbol{y}\right)\right] .
\end{aligned}
$$

Similarly,

$$
\begin{aligned}
P_{e, u}^{(i i)}\left(\boldsymbol{x}_{1}, \boldsymbol{x}_{2}, \boldsymbol{y}\right) & \triangleq \sum_{\left\{\boldsymbol{x}_{1}^{\prime}: U\left(\boldsymbol{x}_{1}^{\prime}, \boldsymbol{x}_{2}, \boldsymbol{y}\right) \geq U\left(\boldsymbol{x}_{1}, \boldsymbol{x}_{2}, \boldsymbol{y}\right)\right\}} Q_{1}\left(\boldsymbol{x}_{1}^{\prime}\right) \\
& =\sum_{\left\{\mathcal{T}\left(\boldsymbol{x}_{1}^{\prime} \mid \boldsymbol{x}_{2}, \boldsymbol{y}\right): U\left(\boldsymbol{x}_{1}^{\prime}, \boldsymbol{x}_{2}, \boldsymbol{y}\right) \geq U\left(\boldsymbol{x}_{1}, \boldsymbol{x}_{2}, \boldsymbol{y}\right)\right\}} \sum_{\tilde{\boldsymbol{x}}_{1} \in \mathcal{T}\left(\boldsymbol{x}_{1}^{\prime} \mid \boldsymbol{x}_{2}, \boldsymbol{y}\right)} Q_{1}\left(\tilde{\boldsymbol{x}}_{1}\right) \\
& \leq \sum_{\left\{\mathcal{T}\left(\boldsymbol{x}_{1}^{\prime}, \boldsymbol{x}_{2}^{\prime} \mid \boldsymbol{y}\right): U\left(\boldsymbol{x}_{1}^{\prime}, \boldsymbol{x}_{2}^{\prime}, \boldsymbol{y}\right) \geq U\left(\boldsymbol{x}_{1}, \boldsymbol{x}_{2}, \boldsymbol{y}\right)\right\}} \sum_{\tilde{\boldsymbol{x}}_{1} \in \mathcal{T}\left(\boldsymbol{x}_{1}^{\prime} \mid \boldsymbol{x}_{2}^{\prime}, \boldsymbol{y}\right)} Q_{1}\left(\tilde{\boldsymbol{x}}_{1}\right) \\
& =\sum_{\left\{\mathcal{T}\left(\boldsymbol{x}_{1}^{\prime}, \boldsymbol{x}_{2}^{\prime} \mid \boldsymbol{y}\right): U\left(\boldsymbol{x}_{1}^{\prime}, \boldsymbol{x}_{2}^{\prime}, \boldsymbol{y}\right) \geq U\left(\boldsymbol{x}_{1}, \boldsymbol{x}_{2}, \boldsymbol{y}\right)\right\}} Q_{1}\left[\mathcal{T}\left(\boldsymbol{x}_{1}^{\prime} \mid \boldsymbol{x}_{2}^{\prime}, \boldsymbol{y}\right)\right] \\
& \leq \sum_{\left\{\mathcal{T}\left(\boldsymbol{x}_{1}^{\prime}, \boldsymbol{x}_{2}^{\prime} \mid \boldsymbol{y}\right): U\left(\boldsymbol{x}_{1}^{\prime}, \boldsymbol{x}_{2}^{\prime}, \boldsymbol{y}\right) \geq U\left(\boldsymbol{x}_{1}, \boldsymbol{x}_{2}, \boldsymbol{y}\right)\right\}} \exp \left[-n U_{1}\left(\boldsymbol{x}_{1}^{\prime}, \boldsymbol{x}_{2}^{\prime}, \boldsymbol{y}\right)\right],
\end{aligned}
$$

and by the same token,

$$
P_{e, u}^{(i i i)}\left(\boldsymbol{x}_{1}, \boldsymbol{x}_{2}, \boldsymbol{y}\right) \leq \sum_{\left\{\mathcal{T}\left(\boldsymbol{x}_{1}^{\prime}, \boldsymbol{x}_{2}^{\prime} \mid \boldsymbol{y}\right): U\left(\boldsymbol{x}_{1}^{\prime}, \boldsymbol{x}_{2}^{\prime}, \boldsymbol{y}\right) \geq U\left(\boldsymbol{x}_{1}, \boldsymbol{x}_{2}, \boldsymbol{y}\right)\right\}} \exp \left[-n U_{2}\left(\boldsymbol{x}_{1}^{\prime}, \boldsymbol{x}_{2}^{\prime}, \boldsymbol{y}\right)\right]
$$

Now,

$$
\begin{aligned}
& \bar{P}_{e, u}\left(R_{1}, R_{2}, n\right) \\
& =\frac{1}{M_{1} M_{2}} \sum_{i=1}^{M_{1}} \sum_{j=1}^{M_{2}} \operatorname{Pr}\left\{\bigcup_{\left(i^{\prime}, j^{\prime}\right) \neq(i, j)} U\left(\boldsymbol{X}_{1}\left(i^{\prime}\right), \boldsymbol{X}_{2}\left(j^{\prime}\right), \boldsymbol{Y}\right) \geq U\left(\boldsymbol{X}_{1}(i), \boldsymbol{X}_{2}(j), \boldsymbol{Y}\right) \mid(i, j) \text { sent }\right\} \\
& \doteq \boldsymbol{E} \min \left\{1,2^{n\left(R_{1}+R_{2}\right)} P_{e, u}^{(i)}\left(\boldsymbol{X}_{1}, \boldsymbol{X}_{2}, \boldsymbol{Y}\right)+\right. \\
& \left.2^{n R_{1}} P_{e, u}^{(i i)}\left(\boldsymbol{X}_{1}, \boldsymbol{X}_{2}, \boldsymbol{Y}\right)+2^{n R_{2}} P_{e, U}^{(i i i)}\left(\boldsymbol{X}_{1}, \boldsymbol{X}_{2}, \boldsymbol{Y}\right)\right\} \\
& \doteq \boldsymbol{E} \min \left\{1, \sum_{\left\{\mathcal{T}\left(\boldsymbol{x}_{1}^{\prime}, \boldsymbol{x}_{2}^{\prime} \mid \boldsymbol{Y}\right): U\left(\boldsymbol{x}_{1}^{\prime}, \boldsymbol{x}_{2}^{\prime}, \boldsymbol{Y}\right) \geq U\left(\boldsymbol{X}_{1}, \boldsymbol{X}_{2}, \boldsymbol{Y}\right)\right\}}\left[2^{-n\left[U_{0}\left(\boldsymbol{x}_{1}^{\prime}, \boldsymbol{x}_{2}^{\prime}, \boldsymbol{Y}\right)-R_{1}-R_{2}\right]}+\right.\right. \\
& \left.\left.2^{-n\left[U_{1}\left(\boldsymbol{x}_{1}^{\prime}, \boldsymbol{x}_{2}^{\prime}, \boldsymbol{Y}\right)-R_{1}\right]}+2^{-n\left[U_{2}\left(\boldsymbol{x}_{1}^{\prime}, \boldsymbol{x}_{2}^{\prime}, \boldsymbol{Y}\right)-R_{2}\right]}\right]\right\} \\
& \doteq \boldsymbol{E} \min \left\{1, \sum_{\left\{\mathcal{T}\left(\boldsymbol{x}_{1}^{\prime}, \boldsymbol{x}_{2}^{\prime} \mid \boldsymbol{Y}\right): U\left(\boldsymbol{x}_{1}^{\prime}, \boldsymbol{x}_{2}^{\prime}, \boldsymbol{Y}\right) \geq U\left(\boldsymbol{X}_{1}, \boldsymbol{X}_{2}, \boldsymbol{Y}\right)\right\}} 2^{-n U\left(\boldsymbol{x}_{1}^{\prime}, \boldsymbol{x}_{2}^{\prime}, \boldsymbol{Y}\right)}\right\} \\
& \leq \boldsymbol{E} \min \left\{1, \sum_{\left\{\mathcal{T}\left(\boldsymbol{x}_{1}^{\prime}, \boldsymbol{x}_{2}^{\prime} \mid \boldsymbol{Y}\right): U\left(\boldsymbol{x}_{1}^{\prime}, \boldsymbol{x}_{2}^{\prime}, \boldsymbol{Y}\right) \geq U\left(\boldsymbol{X}_{1}, \boldsymbol{X}_{2}, \boldsymbol{Y}\right)\right\}} 2^{-n U\left(\boldsymbol{X}_{1}, \boldsymbol{X}_{2}, \boldsymbol{Y}\right)}\right\}
\end{aligned}
$$




$$
\begin{aligned}
& \leq \boldsymbol{E} \min \left\{1, \sum_{\left\{\mathcal{T}\left(\boldsymbol{x}_{1}^{\prime}, \boldsymbol{x}_{2}^{\prime} \mid \boldsymbol{Y}\right)\right\}} 2^{-n U\left(\boldsymbol{X}_{1}, \boldsymbol{X}_{2}, \boldsymbol{Y}\right)}\right\} \\
& \leq \boldsymbol{E} \min \left\{1,2^{-n\left[U\left(\boldsymbol{X}_{1}, \boldsymbol{X}_{2}, \boldsymbol{Y}\right)-\Delta_{n}\right]}\right\} \\
& =\boldsymbol{E}\left\{2^{-n\left[U\left(\boldsymbol{X}_{1}, \boldsymbol{X}_{2}, \boldsymbol{Y}\right)-\Delta_{n}\right]_{+}}\right\},
\end{aligned}
$$

which is of the same exponential order as the lower bound on $\bar{P}_{e, \theta}(R, n)$, and hence $\bar{P}_{e, u}(R, n)$ is exponentially at least as small as $\min _{\theta \in \Theta} \bar{P}_{e, \theta}(R, n)$.

Similarly as in Section 4 , suppose that $U_{0}, U_{1}$ and $U_{2}$ can be uniformly upper bounded by $U_{0}^{\prime}$, $U_{1}^{\prime}$ and $U_{2}^{\prime}$, respectively, and assume that:

$$
\begin{aligned}
\max _{\boldsymbol{y}} \sum_{\boldsymbol{x}_{1}, \boldsymbol{x}_{2}} Q_{1}\left(\boldsymbol{x}_{1}\right) Q_{2}\left(\boldsymbol{x}_{2}\right) 2^{n U_{0}^{\prime}\left(\boldsymbol{x}_{1}, \boldsymbol{x}_{2}, \boldsymbol{y}\right)} & \leq 1 \\
\max _{\boldsymbol{x}_{2}, \boldsymbol{y}} \sum_{\boldsymbol{x}_{1}} Q_{1}\left(\boldsymbol{x}_{1}\right) 2^{n U_{1}^{\prime}\left(\boldsymbol{x}_{1}, \boldsymbol{x}_{2}, \boldsymbol{y}\right)} & \dot{\leq} 1 \\
\max _{\boldsymbol{x}_{1}, \boldsymbol{y}} \sum_{\boldsymbol{x}_{2}} Q_{2}\left(\boldsymbol{x}_{1}\right) 2^{n U_{2}^{\prime}\left(\boldsymbol{x}_{1}, \boldsymbol{x}_{2}, \boldsymbol{y}\right)} & \dot{\leq} 1 .
\end{aligned}
$$

Then, $U_{0}^{\prime}, U_{1}^{\prime}$ and $U_{2}^{\prime}$ can replace $U_{0}, U_{1}$ and $U_{2}$, respectively, in the universal decoding metric, denoted in turn by $U^{\prime}$, and the upper and lower bounds continue to hold with $U^{\prime}$ replacing $U$. The lower bounds on $P_{e, \theta}^{(i)}\left(\boldsymbol{x}_{1}, \boldsymbol{x}_{2}, \boldsymbol{y}\right), P_{e, \theta}^{(i i)}\left(\boldsymbol{x}_{1}, \boldsymbol{x}_{2}, \boldsymbol{y}\right)$, and $P_{e, \theta}^{(i i i)}\left(\boldsymbol{x}_{1}, \boldsymbol{x}_{2}, \boldsymbol{y}\right)$, in terms of $U_{0}^{\prime}, U_{1}^{\prime}$ and $U_{2}^{\prime}$, respectively, are trivial, of course. As for the upper bounds on $P_{e, u^{\prime}}^{(i)}\left(\boldsymbol{x}_{1}, \boldsymbol{x}_{2}, \boldsymbol{y}\right), P_{e, u^{\prime}}^{(i i)}\left(\boldsymbol{x}_{1}, \boldsymbol{x}_{2}, \boldsymbol{y}\right)$, and $P_{e, u^{\prime}}^{(i i i)}\left(\boldsymbol{x}_{1}, \boldsymbol{x}_{2}, \boldsymbol{y}\right)$, we proceed similarly as follows:

$$
\begin{aligned}
P_{e, u^{\prime}}^{(i)}\left(\boldsymbol{x}_{1}, \boldsymbol{x}_{2}, \boldsymbol{y}\right)= & \sum_{\left\{\left(\boldsymbol{x}_{1}^{\prime}, \boldsymbol{x}_{2}^{\prime}\right): U^{\prime}\left(\boldsymbol{x}_{1}^{\prime}, \boldsymbol{x}_{2}^{\prime}, \boldsymbol{y}\right) \geq U^{\prime}\left(\boldsymbol{x}_{1}, \boldsymbol{x}_{2}, \boldsymbol{y}\right)\right\}} Q_{1}\left(\boldsymbol{x}_{1}^{\prime}\right) Q_{2}\left(\boldsymbol{x}_{2}^{\prime}\right) \\
= & \sum_{\left\{\mathcal{T}\left(\boldsymbol{x}_{1}^{\prime}, \boldsymbol{x}_{2}^{\prime} \mid \boldsymbol{y}\right): U^{\prime}\left(\boldsymbol{x}_{1}^{\prime}, \boldsymbol{x}_{2}^{\prime}, \boldsymbol{y}\right) \geq U^{\prime}\left(\boldsymbol{x}_{1}, \boldsymbol{x}_{2}, \boldsymbol{y}\right)\right\}} \sum_{\left(\tilde{\boldsymbol{x}}_{1}, \tilde{\boldsymbol{x}}_{2}\right) \in \mathcal{T}\left(\boldsymbol{x}_{1}^{\prime}, \boldsymbol{x}_{2}^{\prime} \mid \boldsymbol{y}\right)} Q_{1}\left(\tilde{\boldsymbol{x}}_{1}\right) Q_{2}\left(\tilde{\boldsymbol{x}}_{2}\right)\left(104{ }^{-n U_{0}^{\prime}\left(\boldsymbol{x}_{1}^{\prime}, \boldsymbol{x}_{2}^{\prime}, \boldsymbol{y}\right)} \times\right. \\
= & \sum_{\left.\left(\boldsymbol{x}_{1}^{\prime}, \boldsymbol{x}_{2}^{\prime} \mid \boldsymbol{y}\right): U^{\prime}\left(\boldsymbol{x}_{1}^{\prime}, \boldsymbol{x}_{2}^{\prime}, \boldsymbol{y}\right) \geq U^{\prime}\left(\boldsymbol{x}_{1}, \boldsymbol{x}_{2}, \boldsymbol{y}\right)\right\}} \sum_{\left\{\mathcal{T}\left(\boldsymbol{x}_{1}^{\prime}, \boldsymbol{x}_{2}^{\prime} \mid \boldsymbol{y}\right)\right.} Q_{1}\left(\tilde{\boldsymbol{x}}_{1}\right) Q_{2}\left(\tilde{\boldsymbol{x}}_{2}\right) 2^{\left.n U_{0}^{\prime}\left(\boldsymbol{x}_{1}^{\prime}, \boldsymbol{x}_{2}^{\prime}, \boldsymbol{y}\right): U^{\prime}\left(\boldsymbol{x}_{1}^{\prime}, \boldsymbol{x}_{2}^{\prime}, \boldsymbol{y}\right) \geq U^{\prime}\left(\boldsymbol{x}_{1}, \boldsymbol{x}_{2}, \boldsymbol{y}\right)\right\}} 2^{-n U_{0}^{\prime}\left(\boldsymbol{x}_{1}^{\prime}, \boldsymbol{x}_{2}^{\prime}, \boldsymbol{y}\right) \times} \times \sum_{\left(\tilde{\boldsymbol{x}}_{1}, \tilde{\boldsymbol{x}}_{2}\right) \in \mathcal{T}\left(\boldsymbol{x}_{1}^{\prime}, \boldsymbol{x}_{2}^{\prime} \mid \boldsymbol{y}\right)} Q_{1}\left(\tilde{\boldsymbol{x}}_{1}\right) Q_{2}\left(\tilde{\boldsymbol{x}}_{2}\right) 2^{n U_{0}^{\prime}\left(\tilde{\boldsymbol{x}}_{1}, \tilde{\boldsymbol{x}}_{2}, \boldsymbol{y}\right)} \\
\leq & \sum_{\left\{\mathcal{T}\left(\boldsymbol{x}_{1}^{\prime}, \boldsymbol{x}_{2}^{\prime} \mid \boldsymbol{y}\right): U^{\prime}\left(\boldsymbol{x}_{1}^{\prime}, \boldsymbol{x}_{2}^{\prime}, \boldsymbol{y}\right) \geq U^{\prime}\left(\boldsymbol{x}_{1}, \boldsymbol{x}_{2}, \boldsymbol{y}\right)\right\}} 2^{-n U_{0}^{\prime}\left(\boldsymbol{x}_{1}^{\prime}, \boldsymbol{x}_{2}^{\prime}, \boldsymbol{y}\right)}
\end{aligned}
$$


and similar treatments hold for $P_{e, u^{\prime}}^{(i i)}\left(\boldsymbol{x}_{1}, \boldsymbol{x}_{2}, \boldsymbol{y}\right)$, and $P_{e, u^{\prime}}^{(i i i)}\left(\boldsymbol{x}_{1}, \boldsymbol{x}_{2}, \boldsymbol{y}\right)$. This suggests that, in the case where the class $\mathcal{M}$ is based on finite-state machines,

$$
m_{\theta}\left(\boldsymbol{x}_{1}, \boldsymbol{x}_{2}, \boldsymbol{y}\right)=\sum_{i=1}^{n} \theta\left(x_{1, i}, x_{2, i}, y_{i}, s_{i}\right), \quad s_{i+1}=g\left(x_{1, i}, x_{2, i}, y_{i}, s_{i}\right)
$$

and $Q_{1}$ and $Q_{2}$ are uniform distributions within single type classes, one may use $L Z\left(\boldsymbol{x}_{1}, \boldsymbol{x}_{2} \mid \boldsymbol{y}\right)$, $L Z\left(\boldsymbol{x}_{1} \mid \boldsymbol{x}_{2}, \boldsymbol{y}\right)$ and $L Z\left(\boldsymbol{x}_{2} \mid \boldsymbol{x}_{1}, \boldsymbol{y}\right)$ in the relevant places, i.e., the universal decoding metric would be

$$
\begin{aligned}
& U^{\prime}\left(\boldsymbol{x}_{1}, \boldsymbol{x}_{2}, \boldsymbol{y}\right)=\min \left\{\left[\hat{H}_{\boldsymbol{x}_{1}}(X)+\hat{H}_{\boldsymbol{x}_{2}}(X)-\frac{L Z\left(\boldsymbol{x}_{1}, \boldsymbol{x}_{2} \mid \boldsymbol{y}\right)}{n}-R_{1}-R_{2}\right],\right. \\
& {\left[\hat{H}_{\boldsymbol{x}_{1}}(X)-\frac{L Z\left(\boldsymbol{x}_{1} \mid \boldsymbol{x}_{2}, \boldsymbol{y}\right)}{n}-R_{1}\right],} \\
& \left.\left[\hat{H}_{\boldsymbol{x}_{2}}(X)-\frac{L Z\left(\boldsymbol{x}_{2} \mid \boldsymbol{x}_{1}, \boldsymbol{y}\right)}{n}-R_{2}\right]\right\} \text {. }
\end{aligned}
$$

Thus, we observe that our approach suggests a systematic method to extend earlier results to more involved scenarios, like that of the MAC.

\section{Acknowledgment}

Interesting discussions with Meir Feder and Yuval Lomnitz are acknowledged with thanks.

\section{References}

[1] T. M. Cover and J. A. Thomas, Elements of Information Theory, Second Edition, John Wiley \& Sons, New Jersey, 2006.

[2] I. Csiszár, "Linear codes for sources and source networks: error exponents, universal coding," IEEE Trans. Inform. Theory, vol. IT-28, no. 4, pp. 585-592, July 1982.

[3] I. Csiszár and J. Körner, Information Theory: Coding Theorems for Discrete Memoryless Systems, Academic Press 1981.

[4] I. Csiszár and P. Narayan, "Channel capacity for a given decoding metric," IEEE Trans. Inform. Theory, vol. 41, no. 1, pp. 35-43, January 1995.

[5] M. Feder and A. Lapidoth, "Universal decoding for channels with memory," IEEE Trans. Inform. Theory, vol. 44, no. 5, pp. 1726-1745, September 1998. 
[6] M. Feder and N. Merhav, "Universal composite hypothesis testing: a competitive minimax approach," IEEE Trans. Inform. Theory, special issue in memory of Aaron D. Wyner, vol. 48, no. 6, pp. 1504-1517, June 2002.

[7] V. D. Goppa, "Nonprobabilistic mutual information without memory," Probl. Cont. Information Theory, vol. 4, pp. 97-102, 1975.

[8] J. Y. N. Hui, Fundamental Issues of Multiple Accessing, Chap. 4, Ph.D. dissertation, Massachusetts Institute of Technology, 1983.

[9] A. Lapidoth and J. Ziv, "On the universality of the LZ-based noisy channels decoding algorithm," IEEE Trans. Inform. Theory, vol. 44, no. 5, pp. 1746-1755, September 1998.

[10] Y.-S. Liu and B. L. Hughes, "A new universal random coding bound for the multiple access channel," IEEE Trans. Inform. Theory, vol. 42, no. 2, pp. 376-386, March 1996.

[11] Y. Lomnitz and M. Feder, "Communication over individual channels - a general framework," arXiv:1023.1406v1 [cs.IT] 7 Mar 2012.

[12] Y. Lomnitz and M. Feder, "Universal communication over modulo-additive channels with an individual noise sequence," arXiv:1012.2751v2 [cs.IT] 7 May 2012.

[13] N. Merhav, "Universal decoding for memoryless Gaussian channels with a deterministic interference," IEEE Trans. Inform. Theory, vol. 39, no. 4, pp. 1261-1269, July 1993.

[14] N. Merhav, "Universal detection of messages via finite-state channels," IEEE Trans. Inform. Theory, vol. 46, no. 6, pp. 2242-2246, September 2000.

[15] N. Merhav, G. Kaplan, A. Lapidoth, and S. Shamai (Shitz), "On information rates for mismatched decoders," IEEE Trans. Inform. Theory, vol. IT-40, no. 6, pp. 1953-1967, November 1994.

[16] V. Misra and T. Weissman, "The porosity of additive noise sequences," arXiv:1025.6974v1 [cs.IT] 31 May 2012. 
[17] J. Pokorny and H. M. Wallmeier, "Random coding bound and codes produced by permutations for the multiple access channel," IEEE Trans. Inform. Theory, vol. IT-31, no. 6, pp. 741-750, November 1985.

[18] O. Shayevitz and M. Feder, "Communicating using feedback over a binary channel with arbitrary noise sequence," Proc. ISIT 2005, pp. 1516-1520, Adelaide, Australia, September 2005.

[19] N. Shulman, Communication over an Unknown Channel via Common Broadcasting, Ph.D. dissertation, Department of Electrical Engineering - Systems, Tel Aviv University, July 2003. http://www.eng.tau.ac.il/ shulman/papers/Nadav_PhD.pdf

[20] A. Somekh-Baruch and N. Merhav, "Achievable error exponents for the private fingerprinting game," IEEE Trans. Inform. Theory, vol. 53, no. 5, pp. 1827-1838, May 2007.

[21] J. Ziv, "Universal decoding for finite-state channels," IEEE Trans. Inform. Theory, vol. IT31, no. 4, pp. 453-460, July 1985.

[22] J. Ziv and A. Lempel, "Compression of individual sequences via variable-rate coding," IEEE Trans. Inform. Theory, vol. IT-24, no. 5, pp. 530-536, September 1978. 